\title{
Effect of sophrology on perinatal stress monitored by biopyrrin
}

\author{
Michiko Suzuki $^{1}$, Seiji Isonishi ${ }^{2 *}$, Osamu Morimoto $^{3}$, Masahisa Ogawa ${ }^{4}$, Kazuhiko Ochiai ${ }^{1}$ \\ ${ }^{1}$ Department of Obstetrics and Gynecology, Jikei Aoto Hospital, Tokyo, Japan \\ ${ }^{2}$ Department of Obstetrics and Gynecology, Jikei Daisan Hospital, Tokyo, Japan \\ ${ }^{3}$ Oak Clinic for Ms. Hospital, Chiba, Japan \\ ${ }^{4}$ Clinic Ogawa, Gunma, Japan \\ Email: ${ }^{*}$ isonishi@jikei.ac.jp
}

Received 1 May 2012; revised 28 May 2012; accepted 7 June 2012

\begin{abstract}
Aim: Few stress markers are available for women in perinatal period. We introduced biopyrrin (BPn) for monitoring the perinatal stress and investigated how the stress could be regulated. Methods: Study patients consisted of 220 full-term pregnant women between 2006 and 2008. Cases were analyzed based on 1) pattern of delivery, the use of pain control (anesthesia) or hypnotics (Sophrology), 2) maternal complications, and 3) habitual smoking or alcohol consumption. Urinary samples were collected from pregnant women before and after delivery, and from newborn babies. Results: The average level of BPn in pregnant women was $2.61 \pm 0.25 \mathrm{U} / \mathrm{g} \cdot$ Creatinin (Cre) (Mean $\pm \mathrm{SE}$ ). This is significantly higher than age-matched nonpregnant women $(0.79 \pm 0.24 \mathrm{U} / \mathrm{g} \cdot \mathrm{Cre})$. After normal delivery, BPn was significantly elevated to $5.29 \pm 0.57$ $\mathrm{U} / \mathrm{g} \cdot$ Cre $(\mathrm{p}<0.001)$. BPn decreased significantly to $3.41 \pm 0.51 \mathrm{U} / \mathrm{g} \cdot \mathrm{Cre}$ in sophrology assisted delivery and $4.87 \pm 0.53 \mathrm{U} / \mathrm{g} \cdot \mathrm{Cre}$ under epidural anesthesia $(\mathrm{p}$ $<$ 0.05), while delivery induction elevated BPn (11.62 $\pm 1.95 \mathrm{U} / \mathrm{g} \cdot \mathrm{Cre})$. Conclusions: BPn might be an excellent indicator for monitoring perinatal stress. Significant decrease of BPn in sophrology-assisted delivery suggests that the technique should be useful for the relief of perinatal stress.
\end{abstract}

Keywords: Biopyrrin; Oxidative Stress; Sophrology; Anesthesia

\section{INTRODUCTION}

Maternal stress during pregnancy is associated with increased occurrences of preterm birth, developmental delays, low birth weight, birth defects, and an increased risk for neurological and behavioral developmental impairments in the offspring. However, much less is known

"Corresponding author. about the effects of such stress in mothers. The adult rat model is mirroring the human condition: cognitive impairments [1], altered sexual behavior [2], increased anxiety levels [3], and symptoms akin to depression [4]. Researchers of prenatal stress have generally confined themselves to only one aspect of stress or anxiety, such as the effects of life events, work load, or general stress which was evaluated by using self-administered inventories. Physiologic and hormonal responses to stress in pregnancy or the pregnancy-specific anxieties have been sporadically investigated to date $[5,6]$.

On the other hand, lots of data have been reported on the pregnancy-associated oxidative stress. Oxidative stress is enhanced by smoking and alcohol [7], pregnancy-induced hypertension $[8,9]$, and gestational diabetes mellitus [10].

Biopyrrin (BPn) is an oxidative degradation product of bilirubin and is generated under various stresses such as infection, ischemia, and surgery. Stress converts oxygen to activated form of oxygen, which induces hemoxidase activity, a rate-regulating enzyme of bilirubin. Hemoxidase degrades heme to increase bilirubin production. Bilirubin acts as an active oxygen scavenger [antioxidant] and generates BPn. Stocker [11] et al. reported the antioxidant activity of bilirubin, and Shimizu [12] et al. prepared the anti-bilirubin antibody 24G7. An unknown substance that binds to this antibody $24 \mathrm{G} 7$ was found to increase in the urine of patients undergoing abdominal surgery. This substance, an oxidative metabolic product of bilirubin, was designated as biopyrrin. Seven isomers of BPn from X1 to X7 have been confirmed, and their 2 molecular structures have been identified. Since $24 \mathrm{G} 7$ antibody cross-reacts with conjugated bilirubin, the plasma level of BPn could not be measured independently. In this study, urinary BPn was assessed in pregnant women to evaluate whether the various modification techniques for delivery including induction, anesthesia and psychological relaxation have any effects on women's psycho- 
logical status.

Some methods of prenatal childbirth preparation such as Lamaz $[13,14]$, sophrology $[15,16]$, and respiratory autogenic training [17] have been developed in an attempt to decrease anxiety and physical exertion and to improve perinatal outcomes. Sophrology was originally developed by Alfons Caycedo to get the psychological serenity and harmony, spirit and consciousness, by combining Western relaxation therapy and Eastern yoga or meditation [18]. Sophrology for childbirth preparation has gained a good reputation in different countries and has been successfully used in Europe and Korea. In Japan, the technique was first introduced by Dr. Matsunaga (The President of Japan Sophrology Association). He applied sophrology to childbirth preparation combining with guided imagery and music (GIM) which was composed specifically for this medical treatment [19]. Our data showed that sophrology in combination with this music effectively decreased urinary BPn, strongly suggesting that sophrology technique brings the pregnant women psychological relaxation at the perinatal period.

\section{MATERIALS}

Before you begin to format your paper, first write and The BPn level was determined by direct binding ELISA with a BPn EIA Kit, using anti-bilirubin antibody (ALPlabeled 24G7, Shino-test, Japan).

\section{METHODS}

This study was conducted with the approval of the Ethical Review Board of Jikei University School of Medicine. The subjects were prospective cohort of 220 singleton pregnant women who consented to be enrolled between November 2006 and February 2008.

\subsection{Urinary Biopyrrin}

Urinary sample was collected at the following each data point:

1) Anytime before delivery at full term;

2) After the onsets of labor pain;

3) Two to 4 hours after delivery.

Urine samples were stored in the dark at $-20^{\circ} \mathrm{C}$ immediately after collecting. The BPn level was determined by direct binding ELISA and the value was corrected by the urinary creatinine level.

Pregnant women were divided into 3 groups by delivery patterns: spontaneous delivery $(\mathrm{Sp})$, induced delivery (In), and cesarean section (Cs), or by the types of delivery assistant: epidural anesthesia, sophrology, and delivery without any assistance.

Effects of complications of diabetes or hypertension on BPn were analyzed by comparing them with normal pregnant women without any complications.

\subsection{Delivery Control}

The patients were constantly monitored by clinical and tocographic evaluations performed by the staff members of the Department of Obstetrics and Gynecology. The following events were registered and analyzed for purpose of this study:

1) For the patients in induction group, oxytocin administered at a dose level that did not exceed 30 drops/ min of a solution containing 5 units of oxytocin in $1 \mathrm{~L}$ of glucose.

2) Epidural analgesia with bupivacaine was administered for the patients who want delivery under anesthesia.

\subsection{Sophrologic Childbirth Preparation}

Participants hoping for sophrology were trained with sophrologic techniques of relaxation for 4 to 5 hours class during 24 to 28 th week of gestational age. The training in sophrology consisted of two steps. In the first step, pregnant women received the lecture about sophrology by Dr. Morimoto who is now the President of Japan Association of Sophrology. In the second step, pregnant women were trained on the simulating image of delivery process while hearing guided imagery and music (GIM) [19]. During this time, women are trained on deep inspiration and expiration respiratory exercises as well as sophrologic relaxation techniques including the Yoga exercises.

\subsection{Statistical Analysis}

Statistical analysis was performed using two-sample ttest. A two-tailed $\mathrm{p}<0.05$ was considered as the significant. Since there were no previously reported clinical trials evaluating the efficacy of sophrology for decreasing maternal stress monitored by biopyrrin, the sample size was estimated according to the number of pregnant women who were voluntarily enrolled into this study.

\section{RESULTS}

\subsection{BPn in Pregnant Women}

Table 1 summarized the characteristics of pregnant women in each group showing no difference in age, BMI, parity, and gestational age among 3 groups. The overall urinary $\mathrm{BPn}$ level of pregnant women was $2.61 \pm 0.25 \mathrm{U} / \mathrm{g} \cdot \mathrm{Cre}$ (mean $\pm \mathrm{SE}$ ) and was significantly elevated compared with the reported level of historical control in non-pregnant women $(0.79 \pm 0.24 \mathrm{U} / \mathrm{g} \cdot \mathrm{Cre})$ [8]. The level was further elevated to $4.49 \pm 0.37 \mathrm{U} / \mathrm{g} \cdot$ Cre after onset of labor pain or $5.84 \pm 0.48 \mathrm{U} / \mathrm{g} \cdot$ Cre post delivery $(\mathrm{p}<0.001)$. 
Table 1. Characteristics of pregnant women in each group.

\begin{tabular}{|c|c|c|c|c|c|}
\hline \multirow{2}{*}{ Delivery } & \multicolumn{3}{|c|}{ No assistance } & \multirow{2}{*}{ Epidural anesthesia } & \multirow{2}{*}{ Sophrology } \\
\hline & Spontaneous & Induced & Caesarian section & & \\
\hline $\mathrm{N}$ & 56 & 38 & 41 & 13 & 70 \\
\hline Age, years old & $33[18-41]$ & $32.5[18-41]$ & $34[23-41]$ & $28[22-35]$ & $31[19-39]$ \\
\hline BMI & $21.29[17.42-34.29]$ & $22.46[16.45-33.56]$ & $23.81[17.26-34.63]$ & $25.10[21.90-29.78]$ & $24.58[19.68-31.45]$ \\
\hline Para & $1[0-4]$ & $0[0-3]$ & $1[0-2]$ & $0[0-2]$ & $0[0-3]$ \\
\hline Gestational week & $39[37-41]$ & $39[37-41]$ & $38[37-41]$ & $38[37-40]$ & $40[38-41]$ \\
\hline
\end{tabular}

[-]; maximum-minimum.

\subsection{Effect of Delivery Pattern on BPn}

Table 2 listed BPn levels of pregnant women by delivery pattern. On an intra-group comparison, in normal delivery group, BPn levels were mostly constant throughout any phase of delivery. Whereas, in induction or Caesarian group, BPn levels went up significantly after delivery. On an inter-group comparison, post-delivery BPn level of induction group was significantly higher than those of other two groups. The data suggest that delivery induction enhanced maternal psychological stress.

\subsection{Effect of Anesthesia or Sophrology on BPn}

Table 3 listed BPn levels according to delivery assistance. On an intra-group comparison, BPn levels rose with the progress of delivery without regard to the delivery assistance. On an inter-group comparison, BPn levels in any phase of delivery were significantly decreased in anesthesia or sophrology-assisted group compared with those of control group and the effect is even stronger in sophrology group. It is of note that BPn levels before delivery in anesthesia $(2.91 \pm 0.39 \mathrm{U} / \mathrm{g} \cdot \mathrm{Cre})$ or sophrology $(1.28 \pm 0.17 \mathrm{U} / \mathrm{g} \cdot \mathrm{Cre})$ were significantly lower than those in normal delivery $(4.38 \pm 0.51 \mathrm{U} / \mathrm{g} \cdot \mathrm{Cre})$. These data strongly suggested that anesthesia and sophrology reduced psychological stress and the effect is more prominent in sophrology than anesthesia.

\section{DISCUSSION}

Headings, Perinatal depression screening is recognized as an effective means of identifying women at risk for depression during and after pregnancy. The American College of Obstetricians and Gynecologists recommends screening of pregnant and postpartum women for depression, and at least 3 state governments have enacted legislation encourage screening [20-22].

We introduced BPn for quantification and measurement of the degree of psychological status of perinatal women. To the best of our knowledge, this is the first report showing the quantitative analyses of physiological and psychological stress in pregnancy or the effects of pregnancy-specific anxieties. As is expected, pregnant women showed higher level of BPn compared with the population of non-pregnant historical control group, suggesting that even the normally processing pregnant caused the psychological stress. This is well consistent with the report documenting that women are vulnerable to emotional disturbances in response to stress exposure during pregnancy [23]. They are also confronted with other possible stress factors, such as physical alterations and hormonal changes which are often associated with rapid changes in mood and pregnancy-specific anxiety, including fear for child integrity and fear of pain during delivery $[6,24]$.

Normal spontaneous vaginal delivery caused the elevation of BPn and this is more apparent when the delivery was induced while BPn is decreased in the case of caesarean section. There were no previous report pertinent to the psychological status depending on the mode of delivery, however the data in this study suggest that the stronger the delivery pain becomes, the higher the BPn levels go up. In parenthesis, BPn values are not affected by maternal age or duration of pregnancy in our study group. The modes of delivery may have affected psychological status of infants. Recently, it was found that infants born after assisted delivery (forceps or vacuum) showed a greater stress response to inoculation at 8 weeks (they cried more and for longer periods, and had higher cortisol section) than infants born vaginally or by caesarean section [25].

The somatic pain generally causes psychological stress, and the labor pain is one of the stress factors. This is partly confirmed by our data demonstrating that anesthesia-supported delivery and sophrology substantially decreased BPn level. Interestingly, this apparently indicates that psychological status is effectively controlled more by hypnotics than by anesthesia before onset. The results have the positive impact on non-pharmacological treatment options for pregnant women at significant risk of developing postpartum depression, who are generally unwilling to take medication during their pregnancy. The 
Table 2. Effect of delivery pattern on BPn.

\begin{tabular}{|c|c|c|c|c|}
\hline \multirow{2}{*}{ Delivery pattern $[\mathrm{N}]$} & \multirow{2}{*}{ Phase of delivery } & \multirow{2}{*}{$\mathrm{BPn}[\mathrm{M} \pm \mathrm{SE}]$} & \multicolumn{2}{|c|}{ P-value } \\
\hline & & & $\begin{array}{l}\text { Induced delivery/Cesarean } \\
\text { section vs Control }\end{array}$ & $\begin{array}{l}\text { Cesarean section vs Induced } \\
\text { delivery }\end{array}$ \\
\hline \multirow{3}{*}{ Normal delivery [56] } & Before delivery & $5.43 \pm 1.15$ & & \\
\hline & During delivery & $4.86 \pm 0.58$ & & \\
\hline & Post delivery & $5.29 \pm 0.57$ & & \\
\hline \multirow{3}{*}{ Induced delivery [38] } & Before delivery & $5.09 \pm 1.26$ & 0.964 & \\
\hline & During delivery & $7.60 \pm 1.31$ & 0.049 & \\
\hline & Post delivery & $11.62 \pm 1.95$ & 0.003 & \\
\hline \multirow{3}{*}{ Caesarian section [41] } & Before delivery & $3.51 \pm 0.47$ & 0.227 & 0.240 \\
\hline & During delivery & $6.40 \pm 1.00$ & 0.158 & 0.459 \\
\hline & Post delivery & $6.04 \pm 0.91$ & 0.448 & 0.012 \\
\hline
\end{tabular}

BPn; Biopyrrin.

Table 3. Effect of anesthesia or sophrology on BPn.

\begin{tabular}{ccccc}
\hline \multirow{2}{*}{ Delivery assistance [N] } & Phase of delivery & BPn $[\mathrm{M} \pm \mathrm{SE}]$ & \multicolumn{2}{c}{ P-value } \\
\cline { 5 - 5 } & & Control vs Anesthesia/Sophrology & Anesthesia vs Sophrology \\
\hline No assistance [135] & Before delivery & $4.38 \pm 0.51$ & & \\
& During delivery & $6.15 \pm 0.59$ & & 0.03 \\
Anesthesia [13] & Post delivery & $7.39 \pm 0.72$ & 0.537 & 0.008 \\
& Before delivery & $2.91 \pm 0.39$ & $<0.001$ & 0.002 \\
During delivery & $5.22 \pm 1.18$ & $<0.001$ & 0.059 \\
Sophrology [70] & Post delivery & $4.87 \pm 0.53$ & $<0.001$ & 0.065 \\
\hline
\end{tabular}

BPn: Biopyrrin.

data in this study is proposing that sophrology could be effective and useful for the relief of pregnancy associated anxiety particularly for those pregnant women at risk. In this regard, Kim H.H. et al. [16] concluded that they were unable to confirm pregnant women receiving sophrologic childbirth preparation have better maternal clinical outcomes than age- and gestational age-matched control women although they discussed that the psychological benefits of sophrologic childbirth should be explored in future studies. The substantial advantages of our study are to monitor psychological status of pregnant women on digital basis of BPn and to introduce guided imagery and music (GIM) combined with the sophrologic technique. Although the net effect of GIM could not be assessed in our study, it is likely that psychological benefits of sophrology might be explored by BPn and not by the frequency of the use of oxytocin, oxygen, anesthesia or labor induction. One might expect that there are other ways to release physiological stress during delivery. In contrast to sophrology where labor pain is considered as the most indispensable energy in the process of delivering, Lead and Lamaze methods try to obtain an alleviating effect by shutting-off the pain related to labor [26, 27]. However, the Lamaze relaxation technique was not able to decrease distress during labor [28]. One of the trials of non-pharmacological treatments is conducted by Cochrane Pregnancy and Childbirth Group's Trials [29]. Acupuncture was applied to reduce the symptoms of depression; however, it did not significantly decrease the number of women diagnosed with clinical depression 
neither immediately after the treatment nor at final assessment at 10 weeks' postpartum. Our data did not provide the evidence whether sophrology could decrease the onset of clinical depression; however, it is worth noting that BPn level in sophrology has usually started around second trimester of gestation, and evidently this is effective for pregnant women to gain psychological and emotional stability by lowering the level of BPn.

One of the issues we should discuss here is the cause of elevated level of BPn after delivery regardless of the delivery modes or assistant techniques. It is quite natural to expect the sudden decrease of BPn resulted from freedom from delivery anxiety. This inconsistency is probably attributed to the duration of biological reaction and metabolism in which BPn is produced in response to psychological changes.

\section{REFERENCES}

[1] Lordi, B., Patin, V., Protais, P., Mellier, D. and Caston, J. (2000) Chronic stress in pregnant rats: Effects on growth rate, anxiety and memory capabilities of the offspring. International Journal of Psychopsiology, 37, 195-205. doi:10.1016/S0167-8760(00)00100-8

[2] Herrenkohl, L.R. (1986) Prenatal stress disrupts reproductive behavior and physiology in offspring. Annals of the New York Academy of Sciences, 474, 120-128. doi:10.1111/j.1749-6632.1986.tb28003.x

[3] Vallée, M., Mayo, W., Dellu, F., Le, M.M., Simon, H. and Maccari, S. (1997) Prenatal stress induces high anxiety and postnatal handling induces low anxiety in adult offspring: Correlation with stress-induced corticosterone secretion. Journal of Neuroscience, 17, 2626-2636.

[4] Alonso, M.J., Arribas, S., Marín, J., Balfagón, G. and Salaices, M. (1991) Presynaptic M2-muscarinic receptors on noradrenergic nerve endings and endothelium-derived M3 receptors in cat cerebral arteries. Brain Research, 567, 76-82. doi:10.1016/0006-8993(91)91438-7

[5] Van den Bergh, B.R. (1990) The influence of maternal emotions during pregnancy on fetal and neonatal behavior. Pre- and Peri-Natal Psychology Journal, 5, 119-130.

[6] Huizink, A.C. (2000) Prenatal stress and its effects on infant development. Academic Thesis, University Utrecht, Utrecht.

[7] Moller, P., Wallin, H. and Knudsen, L.E. (1996) Oxidative stress associated with exercise, psychological stress and lifestyle factors. Chemico-Biological Interactions, 102, 17-36. doi:10.1016/0009-2797(96)03729-5

[8] Chappell, L.C., Seed, P.T., Briley, A.L., Kelly, F.J., Lee, R., Hunt, B.J., Parmar, K., Bewley, S.J., Shennan, A.H., Steer, P.J. and Poston, L. (1999) Effect of antioxidants on the occurrence of preeclampsia in women at increased risk: A randomised trial. Lancet, 354, 810-816.

[9] Hubel, C.A., McLaughlin, K.M., Evans, R.W., Hauth, B.A., Sims, C.J. and Roberts, J.M. (1996) Fasting serum triglycerides, free fatty acids, and malondialdehyde are increased in preeclampsia, are positively correlated, and decrease within 48 hours post partum. American Journal of Obstetrics \& Gynecology, 174, 975-982. doi:10.1016/S0002-9378(96)70336-8

[10] Toescu, V., Nuttall, S.L., Martin, U., Nightingale, P., Kendall, M., Brydon, P. and Dunne, F. (2004) Changes in plasma lipids and markers of oxidative stress in normal pregnancy and pregnancies complicated by diabetes. Clinical Science, 106, 93-98. doi:10.1042/CS20030175

[11] Stocker, R., Yamamoto, Y., McDonagh, A.F., Glazer, A.N. and Ames, B.N. (1987) Bilirubin is an antioxidant of possible physiological importance. Science, 235, 1043-1046. doi:10.1126/science.3029864

[12] Shimizu, S., Izumi, Y., Yamazaki, M., Shimizu, K., Yamaguchi, T. and Nakajima, H. (1988) Anti bilirubin monoclonal antibody. Preparation and properties of monoclonal antibodies to covalently coupled bilirubin albumin. Biochimica et Biophysica Acta, 967, 255-260. doi:10.1016/0304-4165(88)90017-7

[13] Bernardini, J.Y., Maloni, J.A. and Stegman, C.E. (1983) Neuromuscular control of childbirth-prepared women during the first stage of labor. Journal of Obstetricm \& Neonatal Nursing, 12, 105-111.

[14] Geden, E., Beck, N.C., Brouder, G., Glaister, J. and Pohlman, S. (1985) Self-report and psychophysiological effects of Lamaze preparation: An analogue of labor pain. Research in Nursing \& Health, 8, 155-165. doi:10.1002/nur.4770080209

[15] Lambrette, G. (2000) Sophrology and its relation to child birth [in French]. Soins Pediatr Pueric, 32-35.

[16] Kim, H.H., Nava-Ocampo, A.A., Kim, S.K., Kim, S.H., Kim Y.J., Han, J.Y., Ahn, H.K., Ryu, H.M., Yang, J.H. and Kim, M.Y. (2008) Is prenatal childbirth preparation effective in decreasing adverse maternal and neonatal response to labor? A nested case-control study. Acta Biomed, 79, 18-22.

[17] Cattani, P., Sina, P., Piccolboni, G., Dell'Angelo, M. and Zanarotti, R. (1991) Effect of autogenic respiratory training on labor pain. Use of the Vaona algometer [in Italian]. Minerva Ginecologica, 43, 525-528.

[18] Caycedo, A. (1964) Sophrology and psychosomatic medicine. The American Journal of Clinical Hypnosis, 14, 103106. doi:10.1080/00029157.1964.10402403

[19] Bonny, H.L. (1989) Sound as symbol: Guided imagery and music in clinical practice. Music Therapy Perspectives, 6, 7-10.

[20] Gunderson, E.P. and Abrams, B. (2000) Epidemiology of gestational weight gain and body weight changes after pregnancy. Epidemiology Reviews, 22, 261-274. doi:10.1093/oxfordjournals.epirev.a018038

[21] Kiel, D.W., Dodson, E.A., Artal, R., Boehmer, T.K. and Leet, T.L. (2007) Gestational weight gain and pregnancy outcomes in obese women: How much is enough? $\mathrm{Ob}$ stetrics \& Gynecology, 110, 752-758. doi:10.1097/01.AOG.0000278819.17190.87

[22] Li, C., Goran, M.I., Kaur, H., Nollen, N, and Ahluwalia J.S. (2007) Development trajectories of overweight during childhood: Role of early life factors. Obesity, 15, 760-771. doi:10.1038/oby.2007.585 
[23] Steiner, M. (1979) Psychobiology of mental disorders associated with childbearing. Acta Psychiatrica Scandinavica, 60, 449-464.

doi:10.1111/j.1600-0447.1979.tb00555.x

[24] Nijhuis J.G. and Van den Bergh, B.R.H. (1992) Maternal emotions during pregnancy and fetal and neonatal behaviour. Fetal behaviour. Developmental and Perinatal Aspects, 851, 157-174.

[25] Taylor, A., Fisk, N.M. and Glover, V. (2000) Mode of delivery and subsequent stress response. Lancet, 355, 120. doi:10.1016/S0140-6736(99)02549-0

[26] Delke, I., Minkoff, H. and Grunebaum, A. (1985) Effect of Lamaze childbirth preparation on maternal plasma beta-endorphin immunoreactivity in active labor. American Journal of Perinatology, 2, 317-319. doi:10.1055/s-2007-999979

[27] Mackey, M.C. (1990) Women's preparation for the childbirth experience. Maternal-Child Nursing Journal, 19, 4373.

[28] Bernat, S.H., Wooldridge, P.J., Marecki, M. and Snell, L. (1992) Biofeedback-assisted relaxation to reduce stress in labor. Journal of Obstetric, Gynecologic \& Neonatal Nursing, 21, 295-303. doi:10.1111/j.1552-6909.1992.tb01740.x

[29] Dennis, C.L. and Allen, K. (2008) Interventions [other than pharmacological, psychosocial or psychological] for treating antenatal depression. Cochrane Database of Systematic Reviews, 4, Article ID CD006795. doi:10.1002/14651858.CD006795.pub2 